\title{
A Research on Identify Building Objects from Satellite Images
}

\author{
Manikantan M, Paarivallal Ra,Vakula V
}

\begin{abstract}
Paper presents an innovative Building Object Detection method from the satellite image, which is using the method - Normalized Difference Vegetation Index (NDVI). Remote Sensing and Geographical Information System methods are used frequently in the field of planning and determination of land changes. Recently, Google earth images are used in most of the applications like urban and travel planning etc. The NDVI method is the key aspect to detect the building object automatically. The idea of the proposed method is to detect and identify the building object and periodic changes in the area were detected automatically.
\end{abstract}

KeywordsNormalized Difference Vegetation Index, Google earth images, and Building objects.

\section{INTRODUCTION}

During recent years, building object detection is one of the problems in computer vision and photogrammetric. Buildings are important objects for many applications. Municipal planning, decision support systems, telecommunications, energy potential and efficiency studies, network planning, military applications, aviation purposes, flight simulations, any kind of navigation and mapping applications are all key sectors that require building objects. Telecommunication applications, as an example, require building models to assess and manage the wireless networks. Municipalities and cadastre offices need building models for tax and law purposes. Stability in building information is not always available. Hence, methods are needed to generate building object data.

Earlier, building detection using only image data [1, 2, 3], and some others by using LIDAR data $[4,5,6,7]$. The methods, which use single images, assume that the building objects have simple shape, so the results are very limited. Occlusions and shadows, although a general problem, also cause increased errors, when using one image. In general, the developed methods extract only simple buildings like flat-roof and gable-roof buildings. Secondly, a user interaction is generally required to derive accurate results.

This project variant uses the spectral information classification to detect the buildings. The second variant uses only NDVI and eliminates the trees from the detected objects.

NDVI image extracts the vegetation regions using ISODATA clustering. ISODATA is an unsupervised classification technique, which segments the image into the

Revised Manuscript Received on 14 August, 2019.

Dr. Manikantan M, HoD, Dept. of MCA, Kumaraguru College of Technology, Coimbatore,Tamil Nadu, India. (Email: manikantan.m.mca@kct.ac.in)

Dr. Paarivallal Ra, Assistant Professor / CSE, Bannari Amman Institute of Technology,Tamil Nadu, India.(Email: rpaarivallal@gmail.com)

Ms.Vakula V, Project Assistant, BIT, Sathyamangalam, Tamil Nadu, India.(Email: vakula2596@gmail.com) number of input classes. Finally, the vegetation regions, bare lands, roads and the building objects can be extracted.

In the urban areas lies diverse spectral information, the buildings are to be detected using these variants, red and infrared channels using NDVI. In the LIDAR DTM points to detect the above-ground objects using the density of LIDAR-DTM data. Using the vegetation class from the NDVI classification as a mask removes trees to detect the buildings. The fourth variant is fully based on the LIDAR data, so it does not include any drawbacks from image data (e.g. vegetation on roofs), but has weaknesses, especially on building outlines, which are often detected as trees.

\section{EXISTING METHOD}

Image processing is used to analyze the images through different algorithms. Features are based on characteristics, and indices to highlight the features of interest on the image. Several indices used for highlighting the vegetation areas on a remote area. NDVI is a common and widely used index. The simple numerical indicator NDVI can be used to analyze the remote sensing measurements, from a remote area that contains building objects or not.

Google Earth Engine [8] provides very large satellite images for analyzing the building object, The monocular context is used in methods of region growing, simple geometry models, corners and segments to building detection. In optical remote sensing images, shadow areas are generated with height of building and angle of sun, they produce main clues about building location. The shadow of sides and corner of buildings are used to find the shape and height of the buildings.

To fix the ground control point, and the building information can be extracted from boundary mapping from the aerial images. Additionally, shadow information is used as verification for proposed methods later. The new methods were proposed based on the viewing angle through shadow cast direction. Recently, some classification methods have been proposed to identify and extort buildings automatically using remote sensing imagery.

The Land use maps of an urban area can be extracted from high resolution satellite image like Google Map and this is the base for GIS Application. Existing topographic maps can be updated using the Application. Most of the classification approach used a high-resolution image and spectral image values to extract the buildings. ECHO region-based classifier technique is used to classify the multispectral technique and define the working windows to vectorized the classified images. After that unsupervised 
classification technique, it is used to classify the panchromatic image and also separates building from the background. Finally, building squaring approach is used to depict the boundaries of buildings and it is based on a Hough transform.

\section{STUDY AREA}

The study area for this paper is located in Sathyamangalam, Erode District covering approximately $675 \mathrm{kms}$, the images are extracted from Google maps (high resolution image (sub-meter to 2 meter)). Based on the characters - population density and buildings, it is defined as rural area. Case study on Sathyamangalam, Erode District (6077 sq. km.) of Tamil Nadu lies between the north latitudes $11.5048^{\circ} \mathrm{N}$ and east longitudes of $77.2384^{\circ} \mathrm{E}$ which

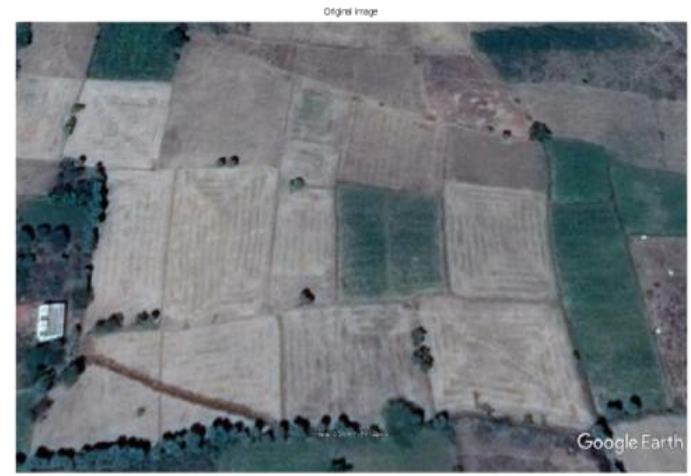

Figure 1: Urban Area Image from Google Earth

\section{PROPOSED METHOD}

The proposed method is one of the building object classification method using Google map image is discussed. The proposed method uses a novel colour invariant with quantization technique for identifying building object. A shadow detection technique is also used. To identify the building regions the entropy segmentation, and watershed segmentation methods are used.

Unsupervised building object identification from an aerial imagery has several advantages. Automatic object detection method has the main advantage to detect object without the user input or parameter adjustment. The assumption employed by the algorithm is considering the building rooftop segments. To detect the building object limited assumptions in different spectral and structural characteristics are used.

The proposed algorithm can classify the objects as classes like building, non building\& vegetation. The accuracy of building object is $97 \%$. The proposed algorithm is highly competitive when compared to other proposed methods. Images taken from Google Earth Engine in two different years 2014 and 2017 for a particular location.

\section{ALGORITHM}

Step 1: Load the image.

Step 2: Construct True Colour Composite from the Image.

Step 3: Use Histograms to Explore Un-Enhanced True colour Composite.

Step 4: Use Correlation to Explore Un-Enhanced True colour Composite. is shown in the Figure. 1

Step 5: Enhance True colour Composite with a Contrast Stretch.

Step 6: Check Histogram Following the Contrast Stretch.

Step 7: Check Correlation Following the De-correlation Stretch.

Step 8: Construct and Enhance a CIR Composite

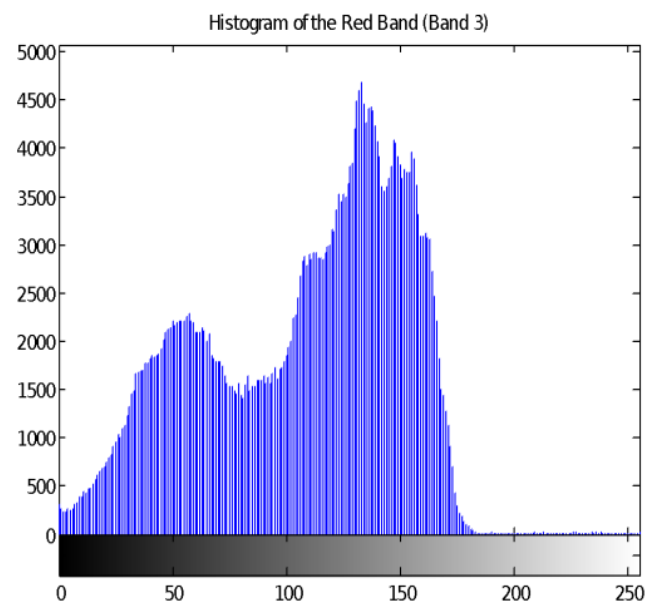

Figure 2: Histogram of the Red Band

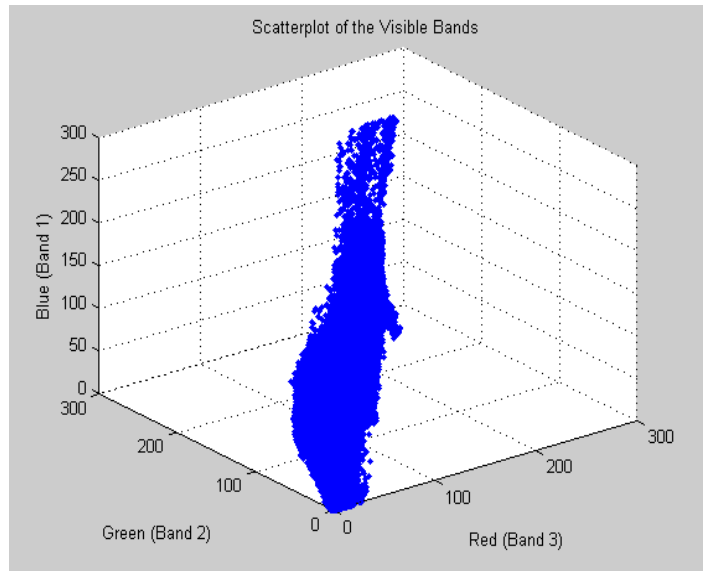

Figure 3: Scatter plot of the Visible Band

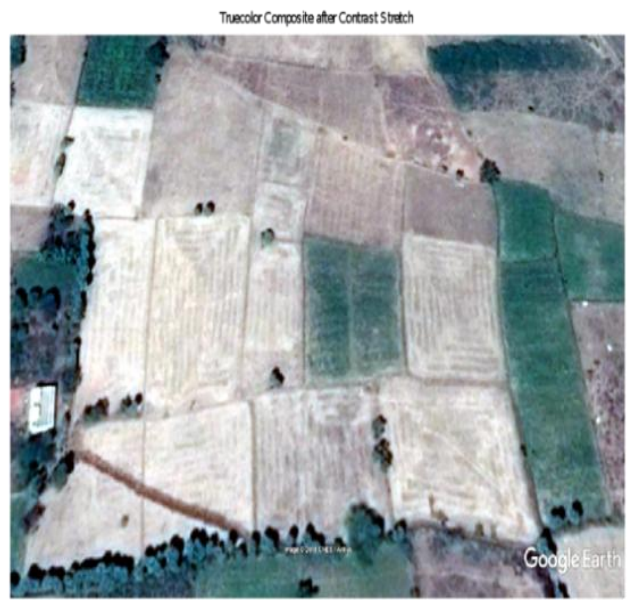

Figure 4: True colour Composite after Contrast Stretch 


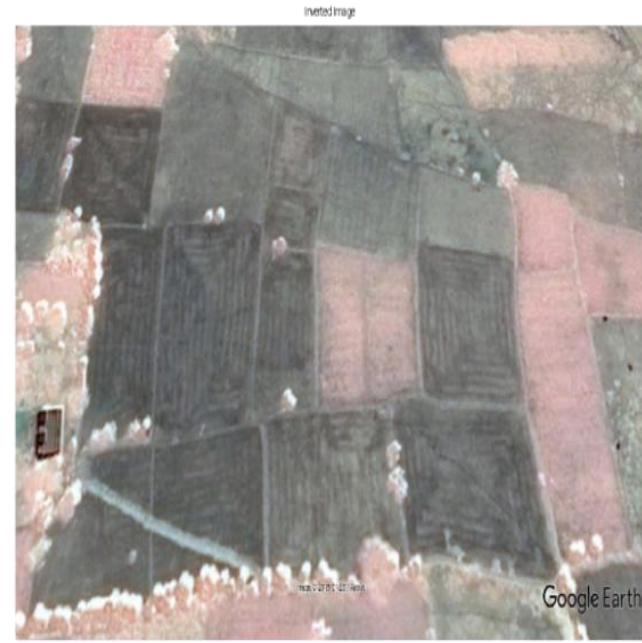

Figure 5: Construct and Enhance a CIR Composite

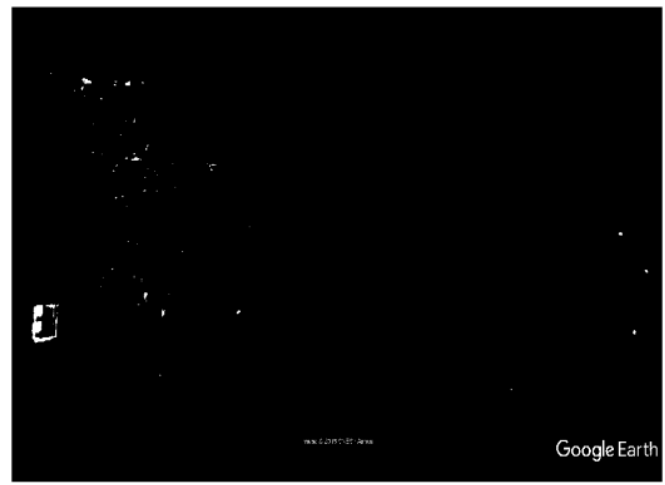

Figure 6 Building object identified

\section{IMPLEMENTATION}

Image classification has an important role in the area of building detection and also in mining analysis in recent years. In Image classification and object diagnosis cluster analysis is used and collection of algorithm is applicable for image processing. However, challenges and difficulties in clustering algorithm is to achieve optimal quality requirements, automation and durability specification [7].

In this proposed method, the NDVI algorithm is integrated to get some solutions. The proposed algorithm determines the analysed result is very efficient in producing cluster and diagnosis of the given data rather than it is useful for object extraction and classification of image.

$$
\mathrm{NDVI}=\frac{\text { NIR-Red }}{\text { NIR+Red }}
$$

The main aim is to implement the identification algorithm to classify the image in an efficient way [1], [4], [8]. They are two steps in image classification, (i) segmentation step and (ii) classification step. In this proposed work, NDVI denotes the Un-Enhanced True colour Composite or Histogram, which is used to segment and classify the image. In this algorithm, there is no specific prerequisite for dataset such as parameters and mathematical details. The algorithm is tested with synthesis dataset and real world dataset.

\section{RESULTS\&CONCLUSION}

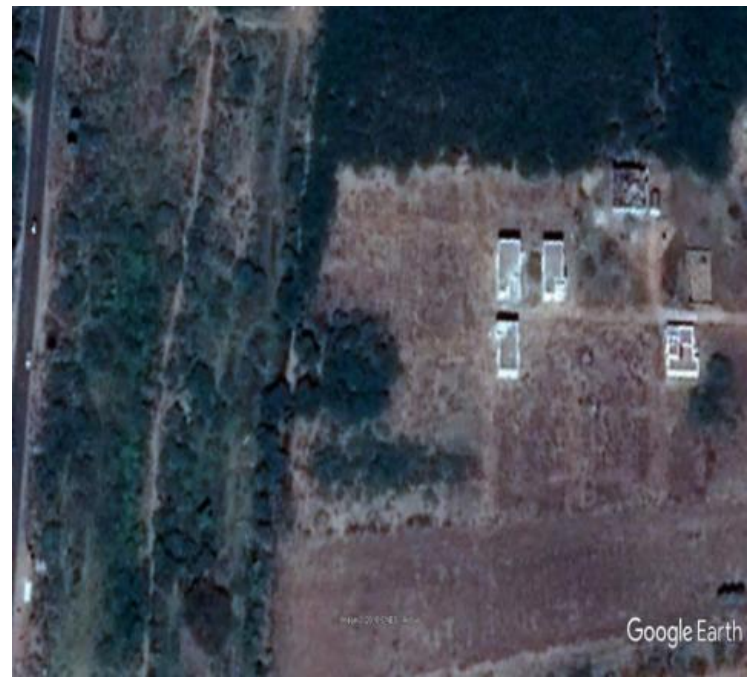

Figure 7(a) original image of 2014

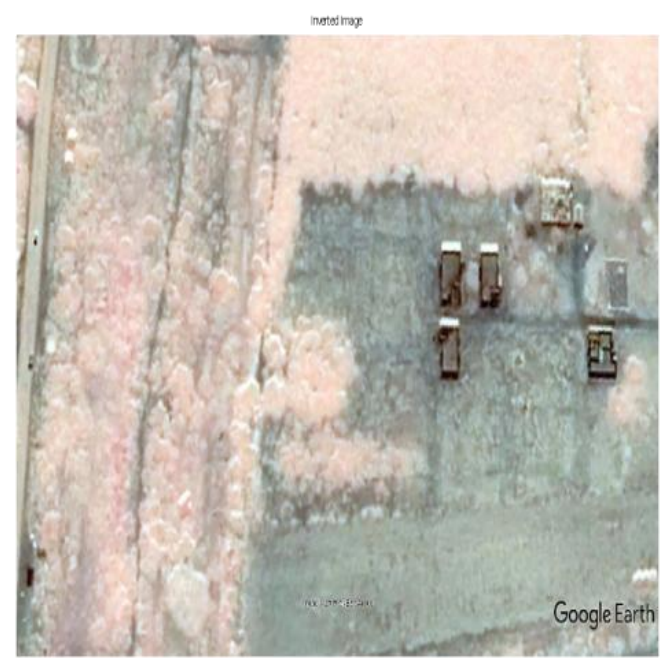

Figure 7(b) output image of 2014

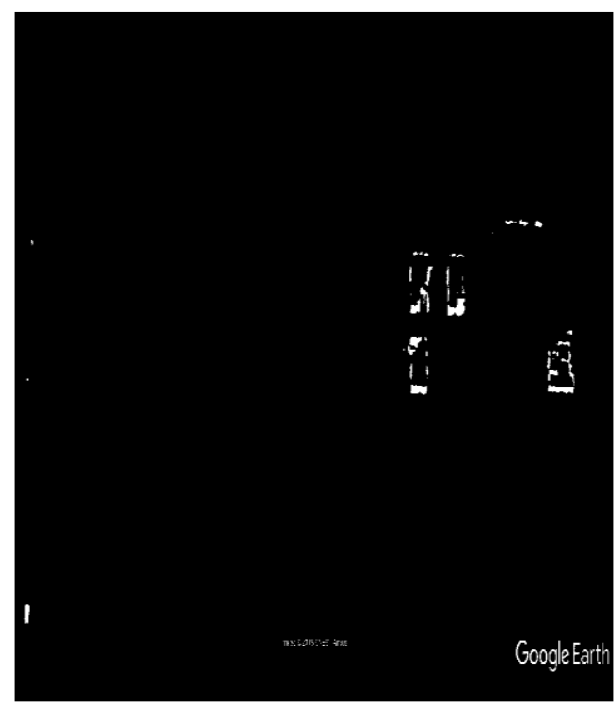

Figure 7(c) Building object identified 


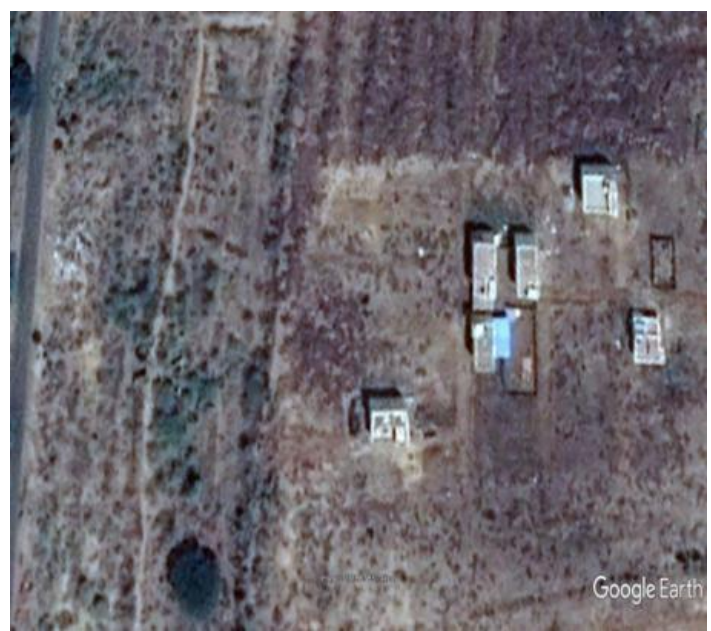

Figure 8(a) original image of 2017

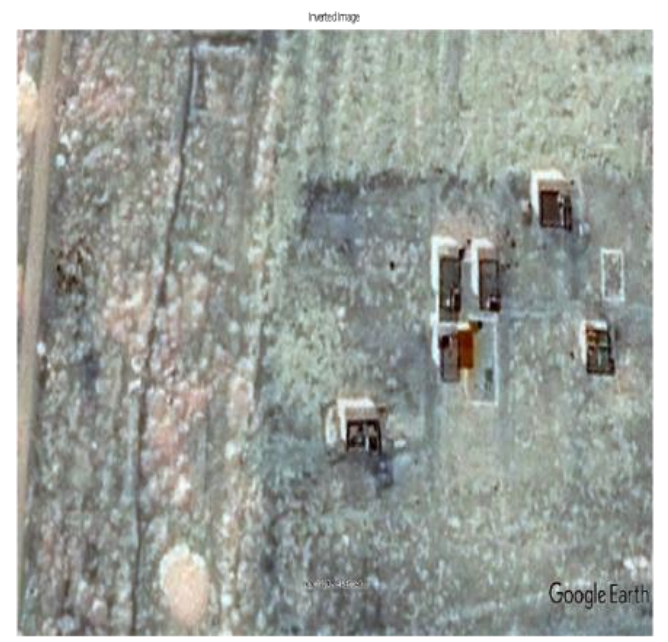

Figure 8(b) output image of 2017

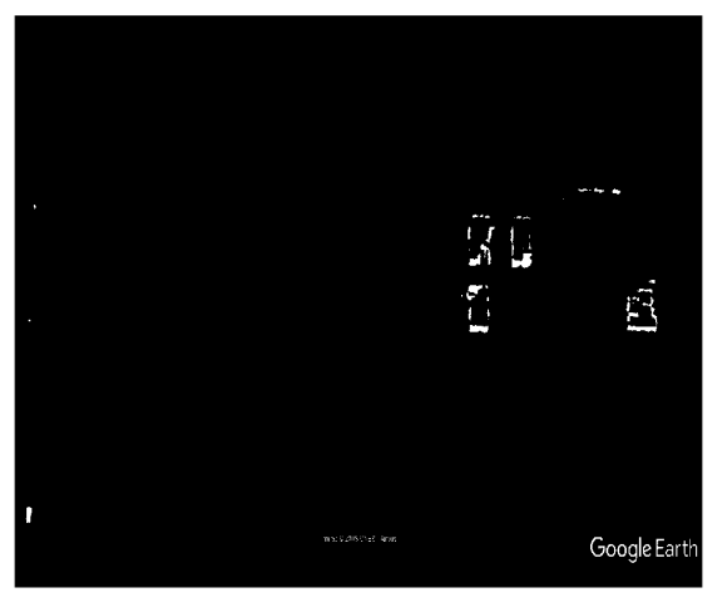

Figure 8(c) Building object identified

To detect the building object, classify the classes into regions like buildings, trees and grass covered areas. Supervised Classification method is used for edge information. The proposed algorithm explain the efficiency to detect colored rooftop buildings. Classification of concrete surfaces such as roads, sidewalks, parking lots, and buildings becomes difficult - especially when those parking lots, sidewalks and roads exist in the sun-side of buildings.
Paper demonstrated the NDVI approach using topographic variables for building objects occurrence in the portion of Sathyamangalam area. In addition to the topographical inputs the proposed algorithm provides best performing model and classification. The proposed method presents an Innovative and novel technique for the object classification and regional planning within the province by taking advantage of recent technological advances and trends in the geospatial sciences.

Proposed method provide the object identification in 92.25\% for Building objects in the image. We have analyzed the two different images in a particular location with two different years 2014 and 2017. This project can easy to find the updated objects in a particular location. This will help to monitor the particular location

\section{REFERENCES}

1. Krüger, T., Meinel, G., \& Schumacher, U. (2013). Landuse monitoring by topographic data analysis. Cartography and Geographic Information Science, 40(3), 220-228.

2. O. Benarchid, N. Raissouni, S. El Adib, A. Abbous, A Azyat, N. Ben Achhab, M. Lahraoua, and A.Chahboun, "Building Extraction using Object-Based Classification and Shadow Information in Very High Resolution Multispectral Images, a Case Study: Tetuan, Morocco, Canadian Journal on Image Processing and Computer Vision Vol. 4 No. 1, January 2013.

3. Aksoy, S., Yalniz, I.Z., Tasdemir, K., 2012. Automatic detection and segmentation of orchards using very high resolution imagery. IEEE Transactions on Geoscience and Remote Sensing 50 (8), 3117-3131.

4. Brenner, C., 2005, Building reconstruction from images and laser scanning, International Journal of Applied Earth Observation and Geoinformation, vol. 6 (3-4), pp 187-198

5. Baltsavias, E.P., 2004, Object extraction and revision by image analysis using existing geodata and knowledge: current status and steps towards operational systems, ISPRS Journal of Photogrammetry and Remote Sensing, vol. 58 (3-4), pp. 129-151.

6. Rajendran, Arunkumar, Nagaraj Balakrishnan, and Mithya Varatharaj. "Malleable fuzzy local median C means algorithm for effective biomedical image segmentation." Sensing and Imaging 17, no. 1 (2016): 24.

7. Y. Wei, Z. Zhao, and J. Song, "Urban Building Extraction from High-Resolution Satellite Panchromatic Image Using Clustering and Edge Detection ," in IGARSS '04, pp. 2008-2010, 2004.

8. K. Segl and H. Kaufmann, 2001,"Detection of small objects from high-resolution panchromatic satellite imagery based on supervised image segmentation," Geoscience and Remote Sensing, IEEE Transactions on, vol. 39, pp. 2080-2083, 2001

9. Google. A Planetary-Scale Platform for Earth Science Data \& Analysis. Available online: https://earthengine. google.com/ (accessed on 29 May 2017)

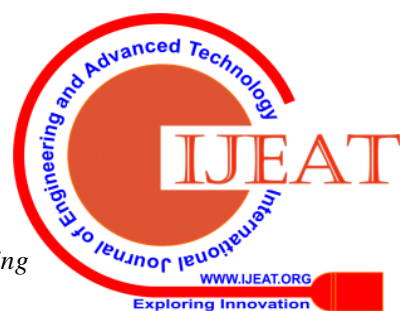

